\title{
FUNCTIONAL STUDY OF WRKY PROTEIN IN DIFFERENT PLANT AND NON-PLANT SPECIES AND ITS RESPONSE UNDER BIOTIC AND BIOTIC STRESSES
}

\author{
Naila Mujahid ${ }^{1}$, Nadia Iqbal ${ }^{1}$, Maryam Zain ${ }^{1}$, Farah Deeba ${ }^{1 *}$ \\ ${ }^{1}$ Department of Biochemistry and Biotechnology, The Women University, Multan, Pakistan \\ Corresponding Author: \\ Dr. Farah Deeba \\ Department of Biochemistry and Biotechnology, The Women University, Multan, Pakistan \\ farah.9003@wum.edu.pk
}

\begin{abstract}
WRKY transcription factors belong to one of the biggest superfamilies of proteins in higher plants. WRKY proteins participate in plant growth for instance, gamete formation, seed germination and are also responsive to different types of environmental cues including abiotic and biotic stresses. The DNA-binding site of WRKY factors is well established which interact with W-box (TGACC(A/T)) located in the promoter of their target genes and promote the activation or repression of the expression of those genes to control their response against stresses but it remains difficult to establish the functions of every family members to control particular transcriptional programs during development or in response to environmental signals. This review summarizes the recent progress made in unraveling the various WRKY protein-controlled functions under different environmental stresses.
\end{abstract}

Key words: Transcription factor, WRKY, stress response. 


\section{INTRODUCTION}

In nature, plants are constantly exposed to various types of environmental stresses at different stages of their life cycle. Two kinds of environmental stresses are faced by plants which can be classified as abiotic stress and biotic stress (1). Abiotic stresses for example drought, flooding, heat and salinity, can cause about $50 \%$ yield loss of major crop plants worldwide (2). On the other hand, attacks by plant pathogens mainly bacteria, fungi, oomycetes, viruses and nematodes are included in biotic stresses $(3,4)$. Plants have no choice to escape from these environmental stresses. To cope with certain abiotic and biotic stresses, plants have evolved different mechanisms. They sense the stress condition, get stimulated and then produce suitable cellular reactions. They do so by receiving stimuli from sensors which are present either on surface of cell or cytoplasm. This signal is then transmitted to the transcriptional machinery located in the nucleus, with the help of various signal transduction pathways by inducing primary genes associated with the stress. This leads to differential transcriptional changes making the plant tolerant against the stress. Subset of these genes include many transcription factors (TFs) like WRKY, ERF, NAC, and MADS. WRKYs are of particular interest as they are involved in diverse biotic/abiotic stress responses as well as in developmental/physiological processes (5). They identify the W-box (TGACC(A/T) present in the promoter of target genes and induce their expression to attain cellular homeostasis. Our goal is to conduct a systematic review to show various contrasting responses can be controlled by a single TF. The emphasis of this review is to highlight the importance of WRKY proteins in regulating various plant processes including plant growth, development and response to various environmental stresses. Moreover, the recent progress made in this area will be highlighted to the best of our knowledge.

\section{Classification and Functional Domains of WRKYs}

WRKY transcription factors are present in large number in plants for instance, 109 in rice and 74 in Arabidopsis. They contain approximately 60 amino acid long four-stranded $\beta$-sheet WRKY DNA binding domain/s (DBD) and Zinc-finger motifs. The WRKY domain is around 60 amino acid residues long, and is capable of binding with DNA $(6,7)$. WRKY proteins are divided into three groups on the basis of presence of WRKY domains and motifs (8). WRKY proteins which possess two WRKY domains are placed in Group I whereas those proteins having one WRKY domain belong to group II or III. Group I and II members have the zincfinger-like motif C2H2 (C-X4-5-C-X22-23-H-X1-H), where $\mathrm{X}$ can be any amino acid residue. 
Group III WRKY proteins contain a C2HC (C-X7-C-X23-H-X-C) zinc-finger-like motif (9). This categorization approach is merely based on characteristics of protein structure and does not include the evolutionary source and gene duplication events. WRKY genes in these groups are not monophyletic and can be further divided into subgroups. For example group II can be organized into five subgroups (II a to II e)on the basis of amino acid sequences $(10,11)$.In addition to WRKY domain, WRKY proteins also include the basic domain of nuclear localization, leucine zippers domain, serine-threonine-rich region, glutamine-rich region, proline-rich region, kinase domain, TIR-NBS-LRR domain and other structures (12). The main WRKYGQK sequence of DNA binding domain shows some variations like WRKYGKK and WRKYGEK $(13,14,15)$.

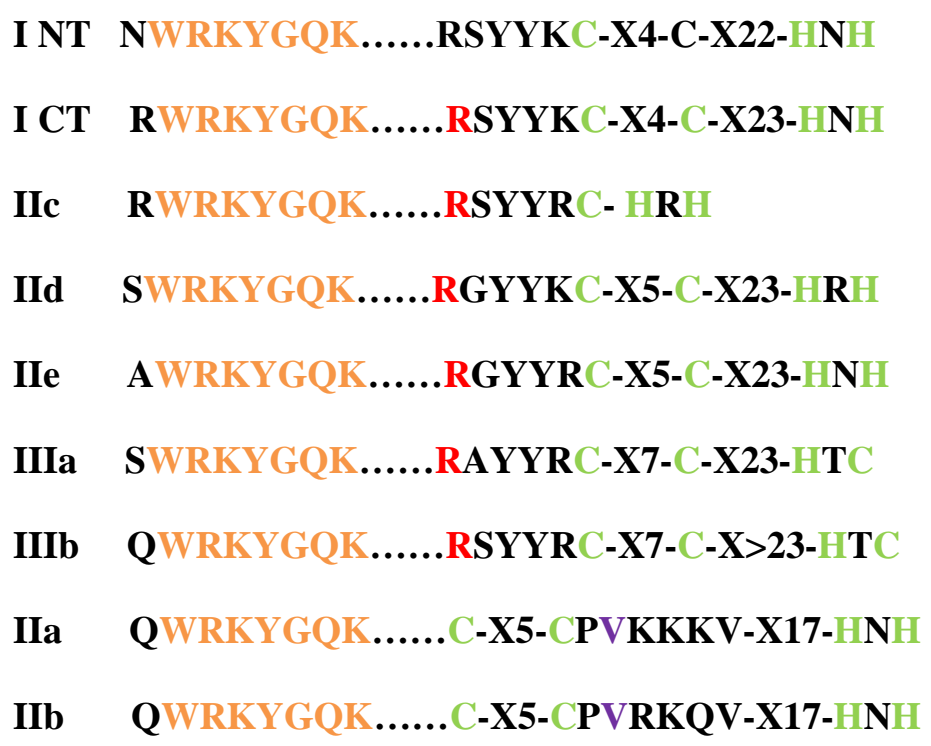

Fig. 1 Scheme of WRKY domain evolution. Representative WRKY domain (WD) of each (sub)group. The consensus amino acid sequences of WDs from rice. The conserved zinc finger in green; the red $\mathrm{R}$ in bold for the conserved R-intron (phase 2), but the R-intron in Chlamydomonasreinhardtii at the position of underlined bold R (ICT line) instead; the purple V in bold for the conserved V-intron (phase 0 ) of subgroups IIa and IIb. One of the introns in I NT of C. reinhardtii is at the position of K, however intronless for rice and Arabidopsis I NT WDs.

WRKY proteins bind with W-box (TTGACC/T) and array of W-boxes located in the promoter region of downstream genes to control the dynamic signaling network by kinase or other phosphorylation cascades. Though WRKYs interact precisely to $\mathrm{W}$ - box, but there are reports of interaction of WRKY proteins to non-W box elements. For instance, OsWRKY13 interacts with both $\mathrm{W}$ box and PRE4 element (TGCGCTT) (16). In the same way, HvWRKY46 (SUSIBA2) can interact to both $\mathrm{W}$ box and a sugar-responsive element (SURE) - 
TAAAGATTACTAATAGGAA (17). On contrary, NtWRKY12 can bind to a SURE-like element but not to the W box sequence. Instead of WRKYGQK, NtWRKY12 has the sequence WRKYGKK and specifically binds to the WK box - TTTTCCAC (17). WRKY DNA binding domain is generally conserved and it binds primarily with W-box motif, nonetheless the stimulation of downstream genes under a particular condition is very specific. This could be due to the presence of motifs and domains exterior of DNA binding domain that offers binding specificity to WRKY proteins under different circumstances. It is perceived that $\beta 1$ and $\beta 2$ sheets of DNA binding domains are typically conserved whereas $\beta 3$ and $\beta 4$ sheets displays inconsistency either in number of amino acids or state of conservation. Thus, binding efficiency of different groups of WRKY to W-box and other motifs seems to be indefinite, which wants additional study and investigation of the domains present outside of DNA binding domains. To characterize the transcription factors and their binding to regulatory elements on genomewide scale, molecular techniques have been developed. For instance, the DAP-seq approach explores genomic DNA with recombinant transcription factor for the identification of transcription factor binding sites (18). Similarly, the ChIP-seq method was effectively employed to discover the binding sites of $A t$ WRKY33 after infecting the Arabidopsis plants with Botrytis cinerea. It was found that AtWRKY33 is involved in negative regulation of abscisic acid (ABA) biosynthesis genes NCED3 and NCED5 (19). The genome-wide investigation of WRKY binding sites also helps us to decode its pleiotropic roles.

\section{Distribution of WRKY genes in the tree of life}

WRKY domains from each of the seven flowering plant groups were studied just as WRKYdomains from one cell containing organism. Furthermore, as WRKY with its WRKY domains are present in many plant and non plant species such as the accompanying species: Arabidopsis thaliana, Glycine max, Brachypodiumdistachyon, S. moellendorffii, P. patens, Chlamydomonasreinhardtii, Chlorella variabilis, Coccomyxasubellipsoidea, Micromonaspusilla, Ostreococcuslucimarinus, Ostreococcustauri, Volvoxcarteri, $K$. flaccidum, Bathycoccusprasinos, Dictyosteliumdiscoideum,Polysphondyliumpallidum, Dictyosteliumfasciculatum, Fonticula alba, Acanthamoebacastellanii, Giardia lamblia, Giardia intestinalis, Dictyosteliumpurpureum, Auxenochlorellaprotothecoides, Spironucleussalmonicida, Mucorcircinelloides, Rhizopusdelemar, Absidiaidahoensis, Lichtheimiacorymbifera, Rhizophagusirregularis, and Mortierellaverticillata. 


\section{Non-plant WRKY genes}

Review of the mushroom WRKY genes show that they are different from plant WRKY genes. The WRKY protein sequences is available as a preserved WKNNGNT as against to WRKYGQK. Parasites contain only one WRKY domain in their WRKY proteins. The studies show that, the ancestral type of WRKY proteins like Division I WRKY transcription factors having two ends (N-and $\mathrm{C}$-terminal) known to be as proto-WRKY having single motif other than space duplication happened (20).As plant-fungi horizontal gene exchanges have all the earmarks of being both uncommon and antiquated (21) this proposes the parasitic sort WRKY qualities may relevant to the first hereditary singular region WRKY gene than complex sub division I quality (22). Amoebae and other amoebozoa are examples of organisms other than plant containing WRKY genes found in division III but they have also resemblance with division I of WRKY gene because they contain 2 WRKY domains (23). Division I WRKY quality have $\mathrm{C}$ region and more than one cell containing PR intron that isolates the WRKY part from $\mathrm{ZnF}$ part. But this intron is absent in organisms that contain only one cell such as $C$. reinhardtii, and $V$. carteri. DivisionI WRKY genes likewise do not have this intron recommending they are similar to green alga (21).

\section{WRKY genes in green algae}

The WRKY genes in unicellular organism such as green algae present in three divisions dependent on sequence study. One group relates to the division I genes found in blossoming plants(24).All complex plant WRKY TFs are descendent of these TFs, to a great extent on the grounds that the main WRKY gene present in the one cell containing organism C. reinhardtii is of this sort. The circumstance in C. reinhardtii may, be that as it may, be a bit of deceiving as some others, for example, $M$. pusilla, $O$. lucimarinus, and $O$. tauri have in excess of a solitary WRKY quality exclude TFs that are members from devisionI (25).

\section{WRKY genes in complex green algae}

As of not long ago, there was great gap between unicellular and multicellular organism according to their patterns in genes, for example, C. reinhardtii that normally have 1-3 WRKY qualities , for example, $P$. patens that have 30-40 qualities. The circumstance altered with the distribution of the $K$. flaccidumgenearrangement(25). There are 2 WRKY qualities in $K$. flaccidum according to gene grouping. The division I quality (kfl00096) having 2 WRKY 
domains like the solelyC. reinhardtii quality. Startlingly, the subsequent gene (kfl00189) is in subdivision IIbquality(26).

similar quality (27).

Division

\section{WRKY genes in flowering plants}

S. moellendorffii contain all group of WRKY genes involve in flowering of plants other than subdivision IIa genes derived from IIb genes. Group IIa genes involve in stress related functions (both biotic and abiotic) but they are less in number (26).

\section{WRKY genes in flowerless green plants}

The primary accessible genome sequencing of flowerless green plant known as moss $(P$. patens) revealed that it has division I, subdivision IIb, subdivision IIc, subdivision IId, and division III I WRKY TFs contain C-end WRKY domain, but $\mathrm{N}$ - terminal domain is not found in Arabidopsis thaliana, parsley (Petroselinum crispum) (29), and sweet potato (Ipomoea batatas) (28).

\section{WRKY TFs in Stress}

Abiotic and biotic stresses negatively affect the plant growth and development which leads to poor crop yield. Such stresses also induce the expression of WRKY transcription factors and generate a web of signaling cascades to develop tolerance against stresses in plants (30). Nevertheless, much less information is available as compared to the research made in deciphering their roles in biotic and abiotic stresses. Despite of discovery of relative large number of WRKY transcription factors from different plants, it remains a great challenge to reveal their roles in different stresses. The strict regulation and fine-tuning of WRKY proteins during plant stress responses contribute to the establishment of complex signaling networks and the important roles of WRKY proteins in plant abiotic stress responses make them potential candidates for introducing stress tolerance (Figure 1). 


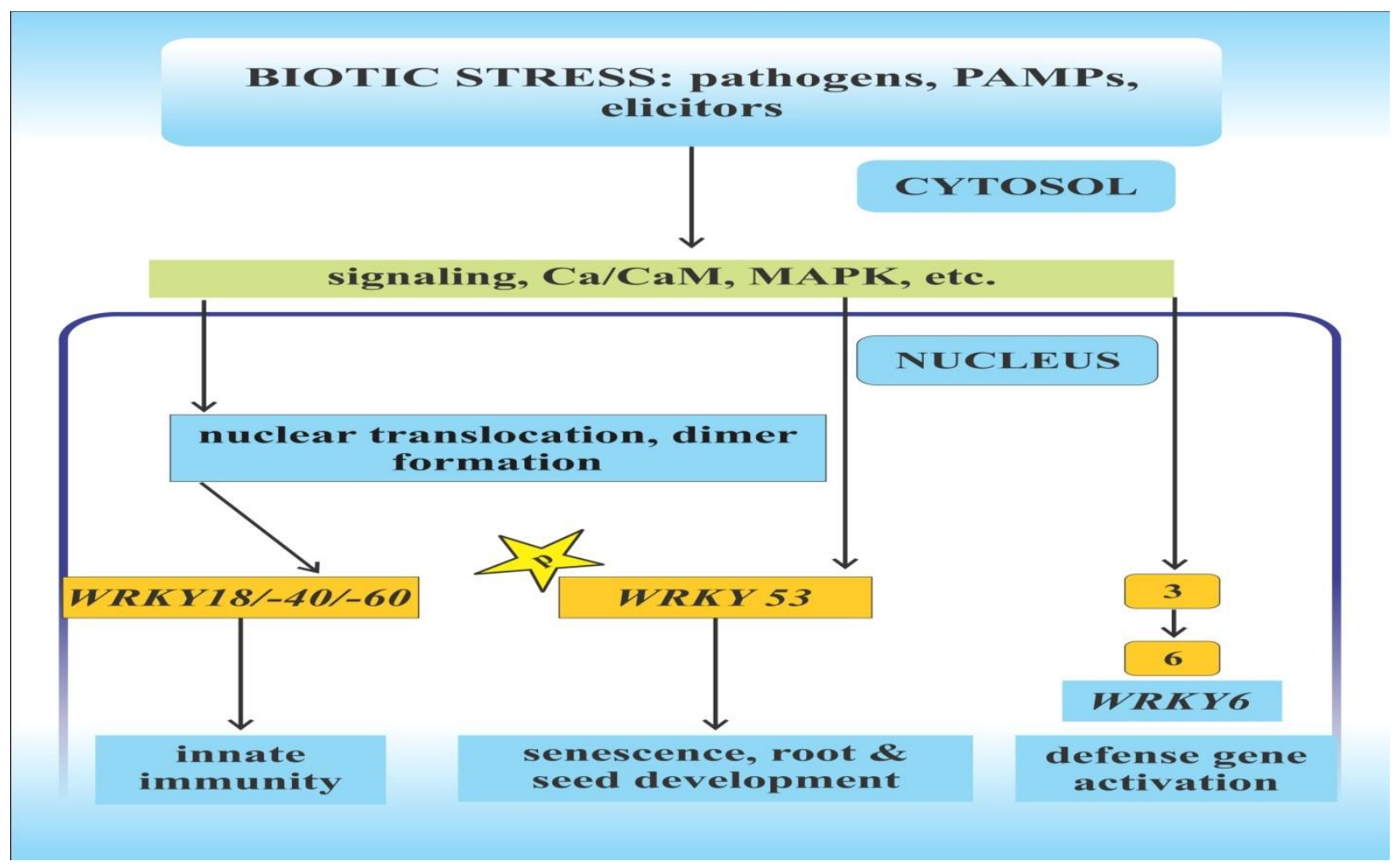

Figure.1: The role of WRKYs and their genes in biotic stress (A), innate immunity (B), senescence root and seed development (C), defense gene activation (D). The yellow square boxes with the number refer to the individual WRKY proteins.

\section{Abiotic Stress}

Transcription factors show their responses opposing to living and environmental stresses. WRKY TFs play their role in opposing environmental stresses and having improved lenience to abiotic stress. The maintenance of WRKY TFs during plant stress reactions causing the improvements in signaling patterns useful for developing the plant strain reactions. Advance report revealed that WRKY TFs having tendency to respond several environmental stresses e.g drought or high salt condition, high and low temperature stress. Advancement in studies revealed that these proteins give more expressions in plants influencing particular strain reactions. Now it is very difficult to relate any particular kind of strain with any specific type of WRKY response. It is hopeful that with the passage of time it might become possible to investigate more or less expression of plants under various strain circumstances and response of the specific WRKY reaction under provided strain condition.

\section{Drought and Salt Stress}

Drought and high salt concentration not only produce uneven characters of salt and water maintenance in the cell yet in addition weaken photosynthesis, energy change in the cell, and 
the oxidation reduction reactions. The control mechanism of WRKY involve signaling of natural circumstances to cell versatile reactions. WRKY TFs are most important regulator for sub-atomic processes to upgrade stress resistance in plants. A few WRKYs are accounted to be associated with drought and saline stress in Arabidopsis, rice, (31) and other plant species. Many built up mechanism components to drought and saline stress are identified with ABA signaling. Very nearly 2000 drought responsive genes were predicted in Arabidopsis under soil drought (32) while some encode for WRKY TFs. There is a discussion among the responses of environmental stresses influences the hormone signaling mechanism. The vast majority of the drought elated genes improved to typical regulation levels about $3 \mathrm{~h}$ succeeding to again watering. Around two third of the dry season genes were additionally managed by WRKY TF genes. Investigation of promoter domains depict that a significant number of the dry spell genes are influenced via ABA signaling. Elevated expression of WRKY57 improved dry spell tolerance of Arabidopsis by elevation of ABA signaling levels (33). ABA signaling and dry spell resistance is controlled by WRKY20 in Arabidopsis (34).GsWRKY20 expressed more ranks and more responsive to $\mathrm{ABA}$ for closing of stomata and demonstrated better lenience in lack of rain, so water deficiency will be less, and opening vesicle denseness will also less (35) due to upregulation of different TFs such as AtbHLH17 and AtWRKY28 in Arabidopsis. The regions of plant that contain genes from different plants appeared to be better lenience to $\mathrm{NaCl}$, crystalline alcohol (mannitol) found in plants, and in stress due to imbalance between free radicals and antioxidants. The stress due to mannitol that are osmolytes and give tolerance against various environmental stresses results in a better origin development in plants that contain specific genes of other plants. In different type of stress conditions many downstream marked genes are upregulated in the more expressing ranks and many promoter regions contain cis acting elements within the sensitive genes. So for the production of heat tolerant plants, WRKY is one of the useful transcription factor. WRKY25 and WRKY33 has enhanced saline lenience and sensitivity to ABA signaling do not rely on the process that express in A.thaliana which is SOS pathway (36).Likewise, to make too many copies of OsWRKY45 caused the improved saline and drought tolerance, and also tolerance against various biotic stresses. ABA induction to GsWRKY20 from natural soybean resulting that this protein useful in lenience of many environmental stresses (37) revealed that WRKY18, - 40 and - 60 are related to each other in different living and nonliving stresses respond by Arabidopsis sprout. The germ and radical growth retardation due to high ABA concentration on plants results in stress response of WRKY18 and WRKY60.Whereas WRKY40 opposes the WRKY18 and WRKY60 in 
influence of $\mathrm{ABA}$ compassion and environmental stresses in development and growth markers. Efficient induction of WRKY18 and WRKY40 through ABA, but WRKY60 is induced by ABA slowly. Cis acting elements are identified by WRKY40 and WRKY18 the activator of WRKY60 and trigger its action in cell. As comparison with WRKY18 and WRKY40 through ABA signaling WRKY60 is regarded marker gene (38).

\section{Cold/Heat Stress}

In Arabidopsis, up and down regulated genes are present such as 655 and 284 respectively and total are 939 (39). Many early cool responsive genes encode for various transcription factors (Figure 2). Especially, the appearance of six WRKY genes (that are induced by cold) was amplified (40) a transformed with affected by low temperature. WRKY is one of the transcription factor that is affected by cold temperature that are present in Arabidopsis (41). WRKY20 is one of the wild type soybean that improves dry season resistance in Arabidopsis and regulates many signaling pathways such as ABA signaling (42).

Adult pollen is exceptionally touchy to cold stress in plants (43) demonstrated that WRKY34 is engaged with pollen feasibility, despite the fact that the definite mechanism is not clear. Chill cure expanded WRKY34 expression in wild sort, and promoter GUS assay, so its pollen specific regulation is exposed.WRKY34 was restricted in nucleus when it is fluorescent labeled protein. Under cold treatment WRKY34 show more feasibility than wrky34 (44).

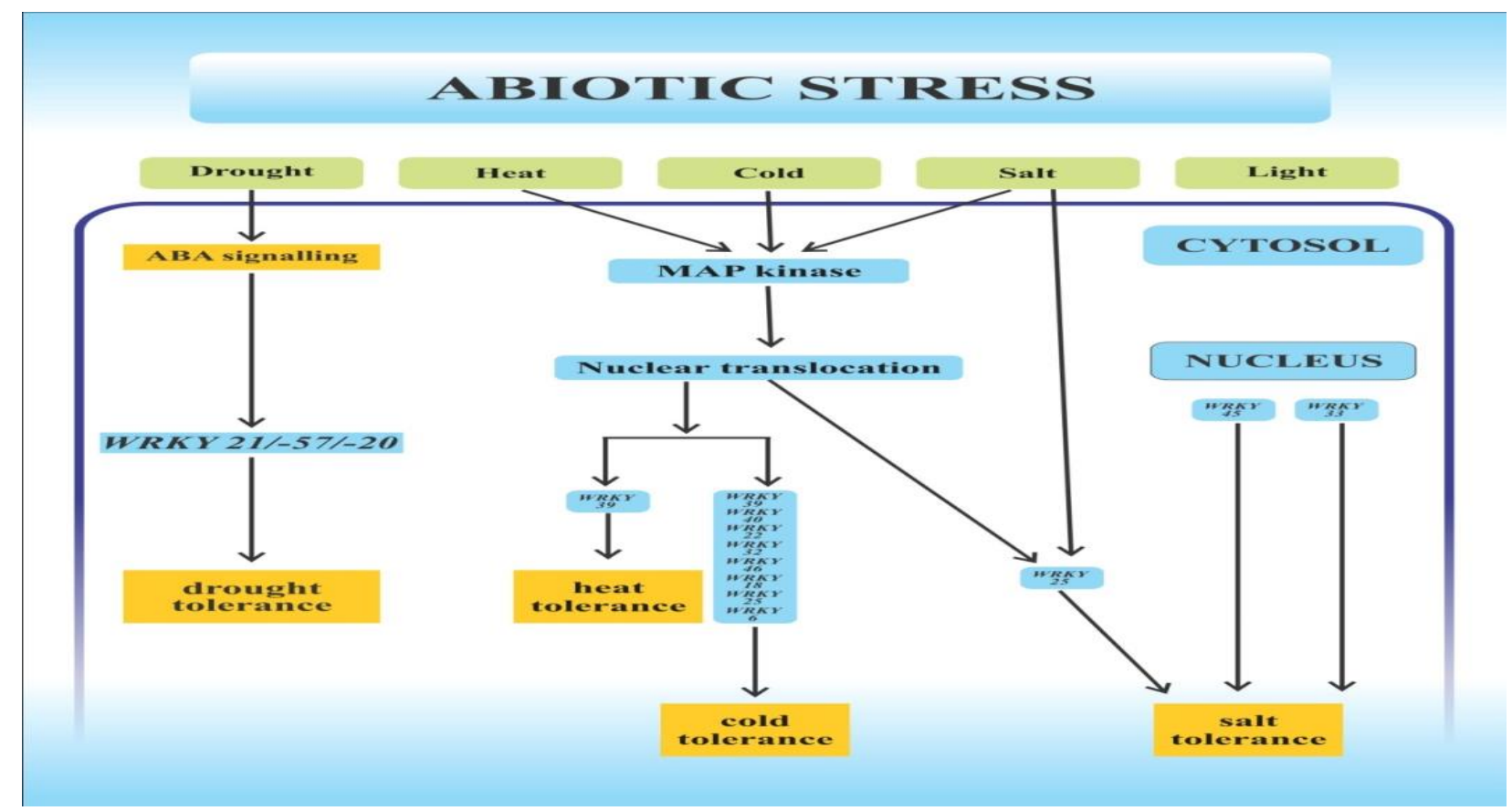


Figure.2: The role of WRKYs and their genes in abiotic stress (A), drought (B), heat tolerance (C), cold tolerance (D), salt tolerance. The blue boxes with the number refer to the individual WRKY proteins.

\section{Results \& Inferences}

In plants WRKY proteins perform different stress resistance, extensive anabolic and catabolic modifications related with maturity, root hair growth, etc. WRKY transcription factors at various levels control gene expression by activation or suppression of genes. Some of WRKY genes like WRKY18 and WRKY40 negatively control EDS1 signaling (45). WRKY25 and 33 are related with environmental stress tolerances, and act as positive and negative regulators of plant defense genes $(21,46$, and 47). Broad investigation of these transcription factor groups is required for improved better understanding of the signaling mechanism involved in plant defense and development. Previously WRKY transcription factors have reported to play role in regulation of plant biotic stresses but recent studies depict their crucial role in abiotic stress tolerance.

These proteins are expressed both under abiotic like high $\mathrm{NaCl}$ and biotic stresses like extreme temperature and pathogen invasion. One of WRKY from Arabidopsis may be used to develop tolerance to various abiotic stresses. Through genetic engineering plants can be transformed with these transcription factors to develop resistance to multi stresses.

\section{REFERENCES}

1. Atkinson, N. J.; Urwin, P. E., The interaction of plant biotic and abiotic stresses: from genes to the field. Journal of experimental botany. 2012,63 (10), 3523-3543.

2. Bray, J. R., Automated language filter. Google Patents: 2000.

3. Strange, R. N.; Scott, P. R., Plant disease: a threat to global food security. Annual review of phytopathology. 2005,43.

4. Montesinos, E., Antimicrobial peptides and plant disease control. FEMS microbiology letters. 2007,270 (1), 1-11.

5. Jiang, Y.; Li, W.; He, X.; Zhang, H.; Jiang, F.; Chen, Z., Lgr5 expression is a valuable prognostic factor for colorectal cancer: evidence from a meta-analysis. BMC cancer. 2015,15 (1), 948 .

6. Yamasaki, K.; Kigawa, T.; Inoue, M.; Tateno, M.; Yamasaki, T.; Yabuki, T.; Aoki, M.; Seki, E.; Matsuda, T.; Tomo, Y., Solution structure of an Arabidopsis WRKY DNA binding domain. The Plant Cel.l 2005, 17 (3), 944-956. 
7.Babu, M. M.; Iyer, L. M.; Balaji, S.; Aravind, L., The natural history of the WRKYGCM1 zinc fingers and the relationship between transcription factors and transposons.

Nucleic acids research 2006, 34 (22), 6505-6520

8.Eulgem, T.; Rushton, P. J.; Robatzek, S.; Somssich, I. E., The WRKY superfamily of plant transcription factors. Trends in plant science. 2000,5 (5), 199-206.

9. Li, C.; Li, D.; Shao, F.; Lu, S., Molecular cloning and expression analysis of WRKY transcription factor genes in Salvia miltiorrhiza. BMC genomics 2015,16 (1), 200.

10. Li, J., Role of WRKY transcription factors in Arabidopsis development and stress responses. Helsinki University Printing House, Helsinki 2014.

11.Chen, L.; Song, Y.; Li, S.; Zhang, L.; Zou, C.; Yu, D., The role of WRKY transcription factors in plant abiotic stresses. Biochimica et BiophysicaActa (BBA)-Gene Regulatory Mechanisms. 2012,1819 (2), 120-128.

12. Chen, L.; Song, Y.; Li, S.; Zhang, L.; Zou, C.; Yu, D., The role of WRKY transcription factors in plant abiotic stresses. Biochimica et BiophysicaActa (BBA)-Gene Regulatory Mechanisms. 2012,1819 (2), 120-128.

13.Jiang, J.; Ma, S.; Ye, N.; Jiang, M.; Cao, J.; Zhang, J., WRKY transcription factors in plant responses to stresses. Journal of integrative plant biology 2017,59 (2), 86-101.

14.Yang, Y.; Zhou, Y.; Chi, Y.; Fan, B.; Chen, Z., Characterization of soybean WRKY gene family and identification of soybean WRKY genes that promote resistance to soybean cyst nematode. Scientific reports. 2017,7 (1), 1-13.

15. Song, H.; Sun, W.; Yang, G.; Sun, J., WRKY transcription factors in legumes. BMC plant biology. 2018, 18 (1), 243.

16.Cai, M.; Qiu, D.; Yuan, T.; Ding, X.; Li, H.; Duan, L.; Xu, C.; Li, X.; Wang, S., Identification of novel pathogen-responsive cis-elements and their binding proteins in the promoter of OsWRKY13, a gene regulating rice disease resistance. Plant, cell \& environment. 2008,31 (1), 86-96.

17. Sun, C.; Palmqvist, S.; Olsson, H.; Borén, M.; Ahlandsberg, S.; Jansson, C., A novel WRKY transcription factor, SUSIBA2, participates in sugar signaling in barley by binding to the sugar-responsive elements of the iso1 promoter. The Plant Cell. 2003,15 (9), 2076-2092. 18.O’Malley, R. C.; Huang, S.-s. C.; Song, L.; Lewsey, M. G.; Bartlett, A.; Nery, J. R.; Galli, M.; Gallavotti, A.; Ecker, J. R., Cistrome and epicistrome features shape the regulatory DNA landscape. Cell. 2016,165 (5), 1280-1292. 
19. Liu, S.; Kracher, B.; Ziegler, J.; Birkenbihl, R. P.; Somssich, I. E., Negative regulation of ABA signaling by WRKY33 is critical for Arabidopsis immunity towards Botrytis cinerea 2100. Elife. 2015,4, e07295.

20.Xie, Z.; Zhang, Z.-L.; Hanzlik, S.; Cook, E.; Shen, Q. J., Salicylic acid inhibits gibberellininduced alpha-amylase expression and seed germination via a pathway involving an abscisicacid-inducible WRKY gene. Plant molecular biology. 2007,64 (3), 293-303.

21. Zhang, Z.-L.; Xie, Z.; Zou, X.; Casaretto, J.; Ho, T.-h. D.; Shen, Q. J., A rice WRKY gene encodes a transcriptional repressor of the gibberellin signaling pathway in aleurone cells. Plant Physiology. 2004,134 (4), 1500-1513.

22.Rinerson, C. I.; Rabara, R. C.; Tripathi, P.; Shen, Q. J.; Rushton, P. J., The evolution of WRKY transcription factors. BMC plant biology. 2015,15 (1), 66.

23.Mohanta, T. K.; Park, Y.-H.; Bae, H., Novel genomic and evolutionary insight of WRKY transcription factors in plant lineage. Scientific reports 2016,6 (1), 1-22.

24. Zhang, Y.; Wang, L., The WRKY transcription factor superfamily: its origin in eukaryotes and expansion in plants. BMC evolutionary biology. 2005,5 (1), 1.

25.Rinerson, C. I.; Rabara, R. C.; Tripathi, P.; Shen, Q. J.; Rushton, P. J., The evolution of WRKY transcription factors. BMC plant biology. 2015,15 (1), 66.

26. Zhao, J.-L.; Pan, J.-S.; Guan, Y.; Nie, J.-T.; Yang, J.-J.; Qu, M.-L.; He, H.-L.; Cai, R., Transcriptome analysis in Cucumissativus identifies genes involved in multicellular trichome development. Genomics.. 2015,105 (5-6), 296-303.

27.Wu, H.; Ni, Z.; Yao, Y.; Guo, G.; Sun, Q., Cloning and expression profiles of 15 genes encoding WRKY transcription factor in wheat (Triticumaestivem L.). Progress in Natural Science. 2008,18 (6), 697-705.

28.Nagata, T.; Hara, H.; Saitou, K.; Kobashi, A.; Kojima, K.; Yuasa, T.; Ueno, O., Activation of ADP-glucose pyrophosphorylase gene promoters by a WRKY transcription factor, AtWRKY20, in Arabidopsis thaliana L. and sweet potato (Ipomoea batatas Lam.). Plant Production Science. 2012,15 (1), 10-18.

29. Agarwal, P.; Reddy, M.; Chikara, J., WRKY: its structure, evolutionary relationship, DNAbinding selectivity, role in stress tolerance and development of plants. Molecular biology reports. 2011,38 (6), 3883-3896.

30. Schluttenhofer, C.; Yuan, L., Regulation of specialized metabolism by WRKY transcription factors. Plant Physiology. 2015,167 (2), 295-306. 
31. Ross, C. A.; Liu, Y.; Shen, Q. J., The WRKY gene family in rice (Oryza sativa). Journal of Integrative Plant Biology 2007,49 (6), 827-842.

32. Dong, J.; Chen, C.; Chen, Z., Expression profiles of the Arabidopsis WRKY gene superfamily during plant defense response. Plant molecular biology 2003,51 (1), 21-37.

33.Huang, S.; Gao, Y.; Liu, J.; Peng, X.; Niu, X.; Fei, Z.; Cao, S.; Liu, Y., Genome-wide analysis of WRKY transcription factors in Solanumlycopersicum. Molecular Genetics and Genomics. 2012,287 (6), 495-513.

34. Ding, Z. J.; Yan, J. Y.; Xu, X. Y.; Li, G. X.; Zheng, S. J., WRKY 46 functions as a transcriptional repressor of ALMT 1, regulating aluminum-induced malate secretion in A rabidopsis. The Plant Journa.l 2013,76 (5), 825-835.

35.Babitha, K.; Ramu, S.; Pruthvi, V.; Mahesh, P.; Nataraja, K. N.; Udayakumar, M., Coexpression of AtbHLH17 and AtWRKY28 confers resistance to abiotic stress in Arabidopsis. Transgenic research. 2013,22 (2), 327-341.

36. Li, J.; Besseau, S.; Törönen, P.; Sipari, N.; Kollist, H.; Holm, L.; Palva, E. T., Defenserelated transcription factors WRKY 70 and WRKY 54 modulate osmotic stress tolerance by regulating stomatal aperture in A rabidopsis. New Phytologist. 2013,200 (2), 457-472.

37.Qiu, Y.; Yu, D., Over-expression of the stress-induced OsWRKY45 enhances disease resistance and drought tolerance in Arabidopsis. Environmental and experimental botany 2009,65 (1), 35-47.

38. Yan, L.; Liu, Z.-Q.; Xu, Y.-H.; Lu, K.; Wang, X.-F.; Zhang, D.-P., Auto-and crossrepression of three Arabidopsis WRKY transcription factors WRKY18, WRKY40, and WRKY60 negatively involved in ABA signaling. Journal of Plant Growth Regulation. 2013,32 (2), 399-416.

39.Chen, L.; Song, Y.; Li, S.; Zhang, L.; Zou, C.; Yu, D., The role of WRKY transcription factors in plant abiotic stresses. Biochimica et BiophysicaActa (BBA)-Gene Regulatory Mechanisms. 2012,1819 (2), 120-128.

40. Li, J.; Brader, G.; Kariola, T.; TapioPalva, E., WRKY70 modulates the selection of signaling pathways in plant defense. The Plant Journal. 2006,46 (3), 477-491.

41. He, G.-H.; Xu, J.-Y.; Wang, Y.-X.; Liu, J.-M.; Li, P.-S.; Chen, M.; Ma, Y.-Z.; Xu, Z.S., Drought-responsive WRKY transcription factor genes TaWRKY1 and TaWRKY33 from wheat confer drought and/or heat resistance in Arabidopsis. BMC plant biology .2016,16 (1), 116. 
42.Luo, X.; Bai, X.; Sun, X.; Zhu, D.; Liu, B.; Ji, W.; Cai, H.; Cao, L.; Wu, J.; Hu, M., Expression of wild soybean WRKY20 in Arabidopsis enhances drought tolerance and regulates ABA signalling. Journal of experimental botany. 2013,64 (8), 2155-2169.

43.Wang, L.; Zhu, W.; Fang, L.; Sun, X.; Su, L.; Liang, Z.; Wang, N.; Londo, J. P.; Li, S.; Xin, H., Genome-wide identification of WRKY family genes and their response to cold stress in Vitis vinifera. BMC plant biology. 2014,14 (1), 103.

44. Zou, C.; Jiang, W.; Yu, D., Male gametophyte-specific WRKY34 transcription factor mediates cold sensitivity of mature pollen in Arabidopsis. Journal of experimental botany. 2010,61 (14), 3901-3914.

45. Zhang, Y.; Wang, L., The WRKY transcription factor superfamily: its origin in eukaryotes and expansion in plants. BMC evolutionary biology .2005,5 (1), 1.

46. Pandey, S. P.; Somssich, I. E., The role of WRKY transcription factors in plant immunity. Plant physiology. 2009,150 (4), 1648-1655.

47. Jiang, Y.; Deyholos, M. K., Functional characterization of Arabidopsis NaCl-inducible WRKY25 and WRKY33 transcription factors in abiotic stresses. Plant molecular biology. 2009,69 (1-2), 91-105. 